\title{
The Growth of Loudness in Cochlear Implant Listeners; Possible Developmental Effects?
}

\author{
Nicole M Anzalone ${ }^{1}$ and Robert L Smith ${ }^{1 *}$ \\ ${ }^{1}$ Institute for Sensory Research and Department of Biomedical and Chemical Engineering, Syracuse University, Syracuse, NY, USA.
}

\begin{abstract}
Received: January 04, 2017; Accepted: February 06, 2017; Published: February 15, 2017
*Corresponding author: Robert L. Smith, Institute for Sensory Research and Department of Biomedical and Chemical Engineering, Syracuse University, Syracuse, NY, USA,13244.E-Mail: rlsmith@syr.edu
\end{abstract}

\begin{abstract}
There have been limited psychophysical measurements on growth of loudness in early-deafened cochlear implant users as compared to postlingually-deafened cochlear implants users. In this study, the "shape" of the loudness growth function was investigated for prelingually- and postlingually-deafened subjects by measuring loudness growth within the electrical dynamic range on different bipolar electrode pairs using Absolute Magnitude Estimation (AME) and Absolute Magnitude Production (AMP). The shapes of the loudness functions were obtained and verified by comparing loudness growth models using the Akaike's Information Criterion (AIC) method to obtain the best- fit functions. Results suggest that adult cochlear implant listeners can exhibit different loudness growth functions, depending on their acoustical experience prior to implantation. Eight subjects with considerable acoustic experience prior to implantation and relatively late implantation demonstrated loudness functions that grew according to an expansive nonlinearity, in agreement with results from several previous studies. In contrast, the four early-deafened subjects with little or no useful acoustic experience prior to implantation, had loudness functions that grew in proportion to stimulus current amplitude. These results suggest that the shape of the loudness function is molded in part by the nature and functionality of input to the central auditory system during auditory development.
\end{abstract}

\section{Introduction}

Loudness relates to the sensation of sound that changes as a function of input magnitude or amplitude. The dynamic range of acoustic hearing can be characterized by the growth of loudness with sound pressure level from the threshold of hearing to the highest intensity sound that one can tolerate before becoming uncomfortably loud. Key findings of previous studies have shown that encoding of magnitude in the auditory system is very different for cochlear implant listeners than it is for acoustic stimulation in normal hearing listeners. In normal acoustic hearing, the loudness growth function generally follows a compressive power law [25] in contrast to electric hearing in which loudness is reportedly an expansive function of applied electrical current, but the specific shape of these expansive functions has not been fully determined. In a previous study by Zeng and Shannon [24], it was suggested that current level in electric hearing is analogous to decibels in acoustic hearing. Therefore, we measured loudness sensation in cochlear implant listening as a function of current level in microamperes.

The growth of sensation magnitude with stimulus intensity is a fundamental property of sensory systems. Beginning with the classical studies by G.T. Fechner and S.S. Stevens, a wide variety of studies in several sensory systems have revealed that the growth of sensation, including loudness, is fundamentally related to the stimulus intensity by a power law. For a power law, $y=a(x)^{b}$ where in normal acoustic hearing $y$ is perceived loudness, $x$ is the sound pressure level (SPL), and b is an exponent approximately equal to 0.6 , (i.e. the function is compressive). However, cochlear implants bypass the auditory periphery and stimulate the auditory nerve directly, and the growth of loudness magnitude with stimulus current for cochlear implant stimulation has been found to be very different from normal hearing individuals. The results typically conclude that loudness grows as an "expansive" nonlinear function of stimulus current. Some studies have shown that loudness growth functions are a power function of current when using magnitude estimation and are best-fit by power functions with an exponent of 2.72 to 3.5 [11,12,30,1,2,18]. Since the exponent range is much higher in electric hearing than acoustic hearing, the dynamic range is much smaller than for normal hearing subjects and hearing-impaired subjects with acoustic stimulation. In modeling the growth of loudness in cochlear implant listeners, there have also been several other proposed models of these expansive functions. The loudness growth functions have also been reported to grow exponentially with stimulus current so that $y=a e^{b x},[30,33]$. Electrical loudness functions have also been described by combinations of both power and exponential functions [16], or by utilizing a forthorder polynomial, which follows the equation $y=a_{0}+a_{1} x^{1}+a_{2} x^{2}$ $+a_{3} x^{3}+a_{4} x^{4}$, [8]. The polynomial fit may be a convenient way to approximate individual variations in how loudness grows with current. While a higher-order polynomial may provide the bestfit approximation of individual variations in loudness functions in electric hearing, higher-order polynomial fits are more sensitive to perturbations or noise in the data.

Previous studies have investigated the relationship between 
loudness growth and the underlying neural mechanisms in processing signals in acoustic hearing and hypothesized that the relationship represents a summation of responses of neural excitation in the peripheral and central auditory system $[31,35]$. Electrical stimulation of the auditory nerve, as in cochlear implant listening, bypasses the peripheral sensory organ to reveal the central processes of the auditory system. A loudness-coding model proposed by Zeng and coworkers [28, $30,31,33$ ] suggest that in the periphery, the normal cochlea provides logarithmic compression. Consequently with the loss of the normal compressive peripheral cochlear mechanics and direct stimulation of the auditory nerve in electrical hearing, an "expansive" exponential nonlinear loudness growth in the central auditory system processing stages is revealed [31]. Similar findings have been described for electrical stimulation of skin [25].

It has been suggested that individual variations in the loudness growth functions may be due to variations in neural processes that accompany cochlear implant stimulation. These variations may be the result of spatial distribution of surviving neurons and/ or contributing neurons, variations in the distributions of electrical fields due to electrode placement, and stimulation mode [15].

Not only can the understanding of the growth of sensation magnitude with stimulus intensity provide a better understanding of neural processes, it also may have clinical implications. Knowing the loudness growth functions in cochlear implant users is presumably necessary for the optimum implementation of the algorithms in the development of speech processors to control overall loudness and the loudness at the level of each electrode. There have been suggestions that the compression algorithm used can affect speech intelligibility [6]. It is also necessary to understand how loudness is encoded and may affect the relative performance in speech intelligibility. Acoustical sound needs to be compressed into the electrical range of hearing in a cochlear implant processor due to the large differences in dynamic range, going from the lowest sound one can hear to the loudest sound one can tolerate, with the electrical dynamic range being much smaller than the acoustic dynamic range (approximately 10:1 for the range of current level in electric hearing, as compared to approximately 1,000,000:1 for the range of sound pressure levels in acoustic hearing). Compression is implemented in the cochlear implant speech processor to fit a larger dynamic range of sounds into the electrical range of hearing and as an attempt to match the logarithmic compression that is missing in the bypassed damaged cochlea. This is based on the current assumption that loudness growth is an exponential function of electrical amplitude and can be predicted exclusively by the threshold and most comfortable levels of loudness, [11, 31]. Having an appropriate loudness growth model is important to cochlear implant users because of the much smaller dynamic range of hearing and distorted loudness growth functions compared to normal hearing listeners [12, 28, 29]. To compensate for this, appropriate models of loudness are necessary for the design of cochlear implant speech processors. Consequently, commercial cochlear implant speech processors currently use a logarithmic function to map acoustic sound pressure to electrical current. Mapping loudness incorrectly from the acoustic sound pressure to electric current may distort speech sounds and reduce speech recognition abilities for cochlear implant users [23, 20, 9, 10, 7]. Findings have shown that speech recognition scores in quiet can drop by a small but clinically significant $8 \%$ to up to $15 \%$ when distorting the compressive function in the speech processor and the best speech recognition performance occurred when the normal loudness function was restored $[1,2,6,12,30]$. Changing the loudness exponent up to a factor of 2 has also been found to have a small effect on speech recognition performance at least in quiet listening conditions, but may have a much greater impact on speech intelligibility performance in noisy listening conditions [12]. In addition, speech intelligibility performance in noisy environments may be detrimentally affected, presumably because environmental noise and background sounds may occupy too much of the already limited dynamic operating range in a cochlear implant.

The major objectives of this study were to examine psychophysical loudness growth measures in twelve adult cochlear implant listeners and to determine whether the Absolute Magnitude Estimation (AME) and Absolute Magnitude Production (AMP) psychophysical methods as previously discussed in Zwislocki and Goodman 1980, can be successfully applied for describing and measuring the sensation of loudness produced by cochlear implants as a function of current level. These methods avoid the presumed bias related to a priori assignment of standards [37].

Currently, there is no consensus on the actual shape of the loudness growth function, with several "expansive" functions being suggested, as described above. The shape of the loudness growth functions measured in cochlear implant users were reexamined based on some a priori models. Since choosing the best-fit models in an objective way can be difficult, Akaike's Information Criterion (AIC, [17]) approach was used as a quantitative way to find the best-fit loudness functions in cochlear implant listeners, and to compare various functions with different numbers of parameters mentioned in previous studies by evaluating goodness of fit. There is a trade-off between goodness-of-fit and parsimony in model selection, but given the limited a priori knowledge, it would seem more appropriate to use a simple model approach. Hence, the AIC method of model selection was used in this study by choosing the best-fit model to the data with the smallest number of parameters.

Some additional goals were to investigate the variation in loudness growth across the population of listeners, for different electrode pairs representing different places along the electrode array, and to investigate the hypothesis that a listener's developmental history may have an effect on the shape of the loudness function. The relationships between electrical dynamic range and threshold values and the shapes of the loudnessgrowth functions were also compared. 


\section{Methods}

\section{Subjects}

Twelve adult CI listeners served as subjects in these experiments. Four of these subjects utilized Cochlear Corporation's Nucleus 22 cochlear implant (subjects S2, S3, S5, S10), seven subjects used a Nucleus 24 cochlear implant (S4, S6, S7, S8, S9, S11, and S12), and one subject (S1) had bilateral implants; a Nucleus 22 on his right side and a Nucleus 24 on his left side. In the case of the bilaterally-implanted subject, measurements were made on each implant separately and results are shown separately as $\mathrm{S} 1 \mathrm{a}$ and $\mathrm{S} 1 \mathrm{~b}$ for the ears with the Nucleus 22 and Nucleus 24 devices, respectively. The subjects ranged from 18 to 70 years of age. Subjects S3, S4, S5, S6, S7, S9, S11, and S12, were postlingually-deafened. Subjects S1a and S1b, S2, S8, and S10 were prelingually-deafened. Patient history and demographic information was obtained before beginning experimentation via a questionnaire and copies of current maps were obtained from subjects' audiologists with permission. Table 1 shows the subjects' demographic information [Table 1].

\section{Stimulus}

Stimuli were delivered through a custom research interface (House Ear Institute Research Instrument: HEINRI) and the NIC I Research Interface. Both systems were calibrated to check consistency for signal delivery. In this study, loudness was directly measured as a function of current level amplitude, as described below.

Signals consisted of trains of $200-\mu \mathrm{sec} /$ phase biphasic pulses with a $20-\mu$ sec silent interval between pulse phases. The biphasic pulse trains were 100-msec-long at a fixed stimulation rate of 500 pps. The stimuli were presented in bipolar $(B P+N)$ mode with varying separation of electrodes. Electrode pair (A, $\mathrm{B})$ therefore indicates current flow between electrode $\mathrm{A}$ and electrode $\mathrm{B}=\mathrm{A}+1+\mathrm{N}$ where the lower numbered electrode, A, serves as the source for the initially negative current pulse and the return is via electrode $\mathrm{B}$. $\mathrm{N}$ represents the number of electrodes between the current source and reference electrode. Electrode pairs were chosen to represent different lengths along the implant arrays, different dynamic ranges, and different

Table 1: Subject demographic information obtained from patient answers on questionnaire.

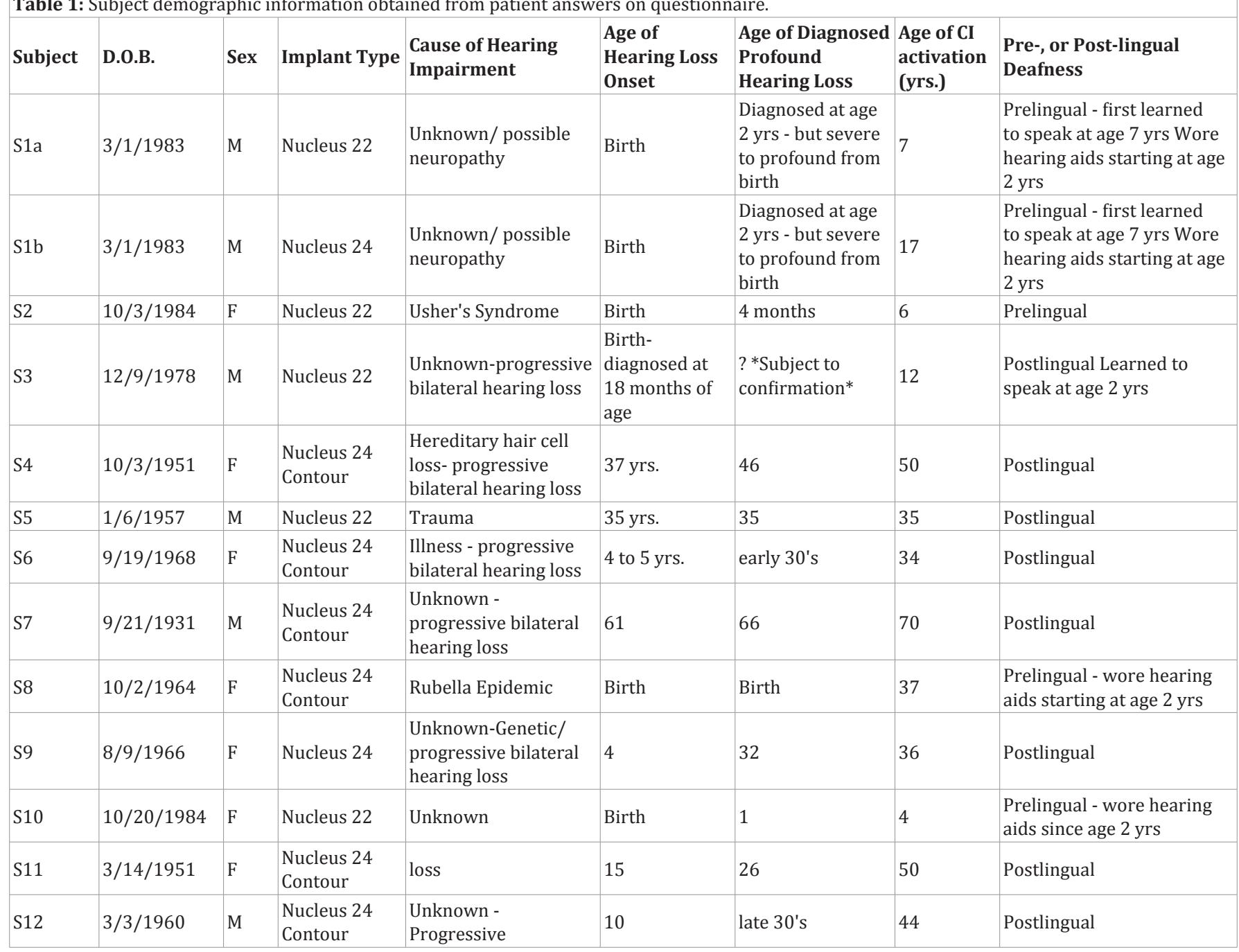


absolute thresholds. Experiments involved presenting stimuli on electrode pairs including; 4,7; 10,13; 16,19; 10,12; 13,16; 12,13; and 10,16 . The most typical bipolar pairs measured were 4,7 ; 10,$13 ; 16,19 ; 10,16$. For precautionary reasons, measurements were not made on electrodes that were flagged or turned off by the subjects' audiologists as indicated by their most recent maps. Effects of varying other stimulus parameters such as stimulus frequency, rate of stimulation, interphase gap, phase duration, and stimulation mode (bipolar, monopolar) were not investigated.

Signal thresholds were approximated either by asking the subject to adjust the current to a just audible level and more precisely by using a 3-interval forced-choice paradigm, with feedback, converging at the $79.4 \%$ correct point [13]. Maximum Allowable Levels (MALs) were determined by asking subjects to adjust the current to the maximum level that they felt was tolerable for experimentation with the particular electrode configuration. Although loudness appeared to play a key role in the choice of MAL, subjects reported that their MAL was also determined by a variety of unacceptable sensations and physiological responses such as facial twitches that would be produced by higher current stimulation. Thresholds, MALs, dynamic ranges (the difference between the MAL and threshold), and loudness growth functions were measured on the above-mentioned bipolar electrode pairs to assess amplitude cues representing various locations across the cochlea. Currents of adjustable magnitude were presented under dedicated computer control, with current changing in steps of one clinical unit when controlled by the subject.

Loudness was measured as a function of current level using the methods of Absolute Magnitude Estimation (AME) and Absolute Magnitude Production (AMP). These methods, based on a wide range of psychophysical studies [36, 25], assume that subjects utilize an internal loudness scale in order to provide numbers or line lengths whose subjective magnitudes match the loudness of the corresponding sounds. The subject's numerical responses are considered a "direct" measurement of their perception of sensation magnitudes. Hence subjective magnitude is assumed to have a single common scale or dimension that is shared by all of these modalities: loudness, numbers, and indeed other sensory magnitudes such as brightness. Furthermore, it is often assumed that loudness is directly proportional to the matching numbers and line lengths, which in turn are approximately proportional to one another. This method has the potential for measuring and comparing loudness under various conditions without utilizing constraints in which particular numbers or line lengths are a priori assigned by the experimenter to particular magnitudes or sensations, hence the term "absolute" is used as opposed to relative which involves a predetermined standard. Standards have been used in the previously cited studies and pre-suppose, for example, that the experimenter can assign the appropriate number for different electrode pairs, i.e. 100 is equated to the MAL. The "absolute" approach attempts to minimize potential biases created by the number range chosen by the experimenter, since such numbers may not necessary correspond to the numbers that the subject would normally use. These biases have been shown to change the shape of the loudness growth curve, for example, increasing the steepness or flattening the curve [37]. In the method of Absolute Magnitude Estimation, the subject is asked to produce a numeric value corresponding to a loudness magnitude he or she perceives based on his or her mental scale, without being given a pre-determined range of numbers to use.

Before beginning the loudness experiments, the subjects were given an initial training session of AME and AMP of line lengths and numbers. Subjects were first asked to match numbers to line lengths, and vice versa, avoiding any explicit reference to upper or lower bounds of numbers. This training or practice session between line lengths and numbers were used to allow the subjects to become more familiar with using their own number scaling system, similar to methods in Collins and Gescheider [5]. Lines of adjustable length were projected on the wall of a dimly lit room against a featureless background. The subjects sat 265 $\mathrm{cm}$ from the wall and observed a thin line projected on the wall at a height of $231 \mathrm{~cm}$. First lines of random length were presented on the wall and the subjects were instructed to assign numbers to match the magnitude of the line length. The subjects were then presented cards with numbers in the range of numbers that were used in the initial training session. The subject could adjust the line length in small steps of approximately $0.2 \%$ of the maximum length of $143 \mathrm{~cm}$ by turning an unmarked Griffin Technology PowerMate® USB Multimedia controller knob connected to our computer-controlled research interface. Results from the practice session revealed that all subjects in this study were able to use their own number scaling to match their perceptual concept of line length effectively and results were generally close to a linear relationship, as found in previous studies [25].

Listeners were then instructed to match loudness of current amplitude stimulation of the cochlear implant to numbers, again using their own natural scales in the AME and AMP experiments. In the AME experiments, the biphasic pulse train signals on a single bipolar electrode pair were presented at different "quasi-random" electrical amplitudes between and including the subject's threshold and MAL for each electrode pair. At least 10 different amplitude levels were presented in a run and each run was repeated three times, with the amplitude levels rerandomized and presented in all three runs. The first run was considered to be a "calibration period" and the geometric mean was taken of only the last two magnitude estimations for each current level and was included in the data analysis, similar to methods in Stevens, 1975.

In the AMP experiments, the subjects were given cards with numbers on them within the range of numbers that they derived in the AME experiments. At least 10 different numbers were quasi-randomly presented in a run and each run was repeated three times, similar to the AME experimental methods. The current level of the biphasic pulses were manipulated by the subject by turning an unmarked Griffin Technology PowerMate ${ }^{\circledR}$ USB Multimedia controller knob connected to our computercontrolled research interface.

In an additional series of experiments, not included in this 
paper, subjects were asked to match line length to loudness and the results appear to closely resemble the experimental results using numbers [21]. Before each of the experiments, the subjects were given a sheet of paper with instructions. Some examples follow:

Absolute Magnitude Estimation: In this experiment, I will present a sound to you. From one presentation to another, the sound may seem different in loudness, at random. Some sounds may seem loud, and others may seem quiet. I am interested in your impression of how loud each sound seems to you. I would like you to assign a number to every sound so that your subjective impression of how large the number is matches your impression of how loud the sound is. You may use any positive numbers that seem appropriate to you - whole numbers, decimals, or fractions. When you think of a number, it may appear subjectively large or small or in between. Obey your intuition and remember that numbers have no limits. There can always be a larger number than the largest you assign and a smaller one than the smallest you assign.

After every presentation of a sound, I would like you to write the number you have assigned on a piece of paper and place it face down in a neat pile on the table next to you. Say "ok" when you are ready for the next presentation. Any questions?

Absolute Magnitude Production: In this experiment, I will give you cards, one at a time, with a number on each. During every card selection, I would like you to adjust the loudness of the sound using the unmarked knob, to match your subjective impression of the magnitude if the number on the card. After you have adjusted the loudness of the sound to match your subjective impression of the magnitude of the number, just say "ok," and I will move on to another card. Any questions?

\section{Data Analysis}

The loudness growth data were obtained using both AME and AMP methods and plotted on linear axes as a function of current level in microamperes.

KaleidaGraph ${ }^{\text {TM }}$ graphical software was used to produce all of the graphical presentation of the figures in this paper and GraphPad Prism version 4 statistical trial software package, San Diego, C.A., was used for curve-fitting and the AIC analyses. SPSS ${ }^{\circledR}$ version 15 (SPSS, Inc.) was used specifically for statistical analysis. A variety of models were used, as per those used in earlier studies, these include some of the following and a straight line was also used based on visual inspection.

\section{Linear Function Model (LFM)}

$\mathrm{L}=\mathrm{ax}+\mathrm{k}$

\section{Power Function Model (PFM)}

$\mathrm{L}=\mathrm{a}(\mathrm{x})^{\mathrm{b}}+\mathrm{k}$

\section{Exponential Function Model (EFM)}

$\mathrm{L}=\mathrm{a}^{*} \mathrm{e}^{\mathrm{bx}}+\mathrm{k}$

\section{Fourth-order polynomial model}

$\mathrm{L}=\mathrm{a}_{0}+\mathrm{a}_{1} \mathrm{x}^{1}+\mathrm{a}_{2} \mathrm{x}^{2}+\mathrm{a}_{3} \mathrm{x}^{3}+\mathrm{a}_{4} \mathrm{x}^{4}$
The above functions were fit to the loudness data using nonlinear regression and best-fit functions were derived for each individual loudness function using AIC [17], which utilizes a combined maximum likelihood and information theoretical approach and was used to determine the best fit function by determining how well the curve fits the data. In this study, the AIC method of best-fit model selection was used for several reasons. AIC values can be used to compare and best-approximate nonlinear data curves.

Correlation coefficients for models or functions may have close $\mathrm{R}^{2}$ values (i.e. 0.90 vs. 0.92 ) and there is no way to quantify differences in the correlation coefficients for different "best-fit" functions because $\mathrm{R}^{2}$ has no units and only reveals the amount of variability in the data that can be explained by the model [17]. Functions with the highest number of parameters may automatically have higher $\mathrm{R}^{2}$ values because more parameters can better fit fluctuations or noise in the data curves. The least squares method using F-test or P-values for significance can tell you what is significant or not significant or that the models may be statistically different, but cannot tell you which model to use by a direct comparison between two or more models. F tests should only be used for nested models (meaning one function is a simpler case of the other as in multiple polynomial functions) and the F-test cannot be extended to compare three of more models because multiple $\mathrm{P}$ values cannot be directly compared [17]. We used a modified AIC method to compare best-fit growth of loudness functions, called AICc, which is a variation of the AIC equation that utilizes a second term that takes into account the number of parameters [17]. The AICc formula is recommended to use when the number of data points is small relative to the number of parameters. The AICc can be computed as follows;

where $\mathrm{N}$ equals the number of data points, SS is the sum of squares of the vertical distances of the points from the curve or the residuals, $\mathrm{K}$ is the number of parameters fit by the regression plus 1 [17]. If the $\mathrm{N} / \mathrm{K}$ ratio is larger than 40, the second term becomes negligible. The AICc method of best-fit model comparison has been used previously to investigate nonlinear psychophysical modeling [19] and is commonly used for analysis of biological system model comparisons. Comparing AIC values is a good method for comparing non-nested nonlinear models and for evaluating the strength of evidence of the probability that one model is better at approximating the loudness data than the other comparison models or growth functions. The AIC method estimates which function or model most likely best approximates the data and quantifies how much more likely based on the function with the smallest number of parameters without overfitting or underfitting the data [17]. The AIC method can also be used for goodness-of-fit approximation by estimating the relative distance between the fitted model and the data, using a combination of a maximum likelihood method and information theory approach to minimize the residuals [17].

The best-fit growth of loudness function can be determined by comparing the AICc values once fit to the data points and choosing the model with the lowest AICc value. The differences between the AICc values between two models can also help 
explain how the best-fit model can be selected. The differences between the AICc values were also calculated and quantified by approximating the strength of evidence with the evidence ratio of probabilities that one model is better at approximating the data than the other models in comparison. AICc was used to find the model that correctly best fits the loudness growth function data, and was used to quantify how much more likely it fit the data than another comparison models by comparing the output range of probabilities of one model fit compared to the others. The AICc values were computed for all of the possible model comparisons, (i.e. linear vs. power; power vs. exponential; linear vs. exponential, etc.), for each data set or loudness growth curve and the model with the lowest AICc value was selected as the "best-fit" model between the model comparison cases.

\section{Results}

Individual loudness growth functions were obtained for each bipolar pair using loudness measurements by AME and AMP. Subjects produced functions that appeared to fit into two general classes; "expansive" shown in figure 1 and "linear" shown in figure 2. Figure 1 shows the loudness estimates for both AME and AMP, measured on various electrodes for "expansive" group of subjects. For these individuals (Subjects S3, S4, S5, S6, S7, S9, S11, S12), as most frequently encountered in previous studies, loudness appears to grow as an "expansive" (power or exponential) function of applied current, as can be seen by visual inspection of the plots (Figure 1).

Subjects were given the freedom to use any range of numbers corresponding to loudness with the AME procedure,

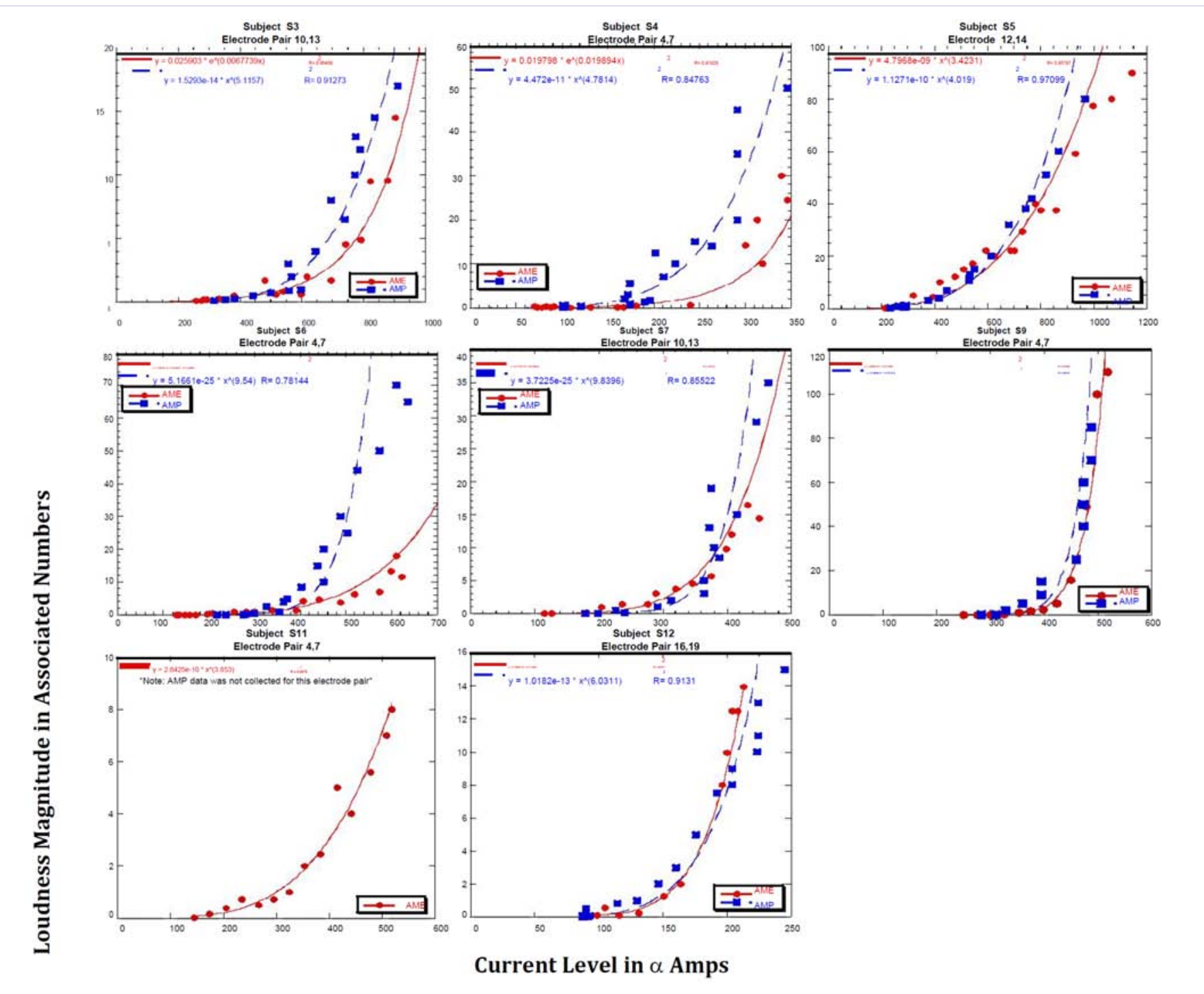

Figure 1: Examples of "expansive" loudness growth functions obtained using Absolute Magnitude Estimation (AME) and Absolute Magnitude Production (AMP) for different subjects. Loudness in associated numbers is plotted versus current applied to the designated bipolar electrode pairs. Note AMP was not measured on Subject S11. 
and as shown in figure 1, individuals used different maximum numbers to represent loudness. Figure 1 also shows that AME and AMP procedures produced similar results, as illustrated by the closeness in the data points from both experiments. There were a few cases for an individual where the loudness functions for the AME and AMP experiments diverged, demonstrating a slight change in the ranges used by the same subject, as seen for subjects S4, and S6.

In contrast, the loudness functions in figure 2 illustrate that for this second set of individuals (Subjects S1, S2, S8, S10), both AME and AMP yield "linear" functions for the electrode pairs utilized. Results from Subject 1 demonstrated a linear relationship between stimulating current and loudness with both the subject's Nucleus 22 and Nucleus 24 implants, represented by Subject $1 \mathrm{a}$ and Subject $1 \mathrm{~b}$ data (Figure 2).

Visual inspection of loudness growth curves as in figures 1 and 2 appeared to reveal at least two classes of subjects; those that produced the previously seen "expansive" loudness growth functions and those that produced "linear" functions when plotted as a linear function of current level in microamperes. These particular loudness growth functions were well-fit by either exponential (Fig. 1), linear functions (Fig. 2) as evidenced by their correlation coefficients.

\section{Results of the AICc- Best Fit Loudness Growth Functions}

For other subjects/electrode pairs, loudness growth could seemingly be fit with several different functions by visual inspection or by looking at the correlation coefficients alone. Figure 3 is an example of how loudness estimates for one subject could be fit with different models and that by comparing the correlation coefficients and through observation, it is sometimes difficult to classify the best-fit function, consistent with our previous data [21]. For this reason, the AICc analysis was useful in quantifying the best-fit functions in a more objective manner (Figure 3).

Tables 2 and 3 some results of AICc best-fit functions analysis of loudness growth functions measured by Absolute Magnitude Estimation on different electrode pairs for the postlinguallydeafened subjects. Many of these functions were included in the plots in figure 1. It should be noted that high-degree polynomials were unstable and diverged outside of the data range, making it difficult to fit the data, so that data was not included here. The best-fit function was chosen by selecting the model with the lowest AICc values between the linear, power, and exponential fits. The accuracy of the fits for each loudness growth data set can be obtained by pairing two models and finding the AICc difference and comparing the probability between each of the three models based on the difference in AICc values, resulting in the percent likelihood that each model is the correct fit. The percentages, or Akaike's weights, were calculated using the relative likelihood method described by Motulsky \& Christopoulos, [17]. The last column of Table 2 lists the power fit function exponent for each data set and the results are discussed in further detail below. Note that in some individuals, both power and exponential best-

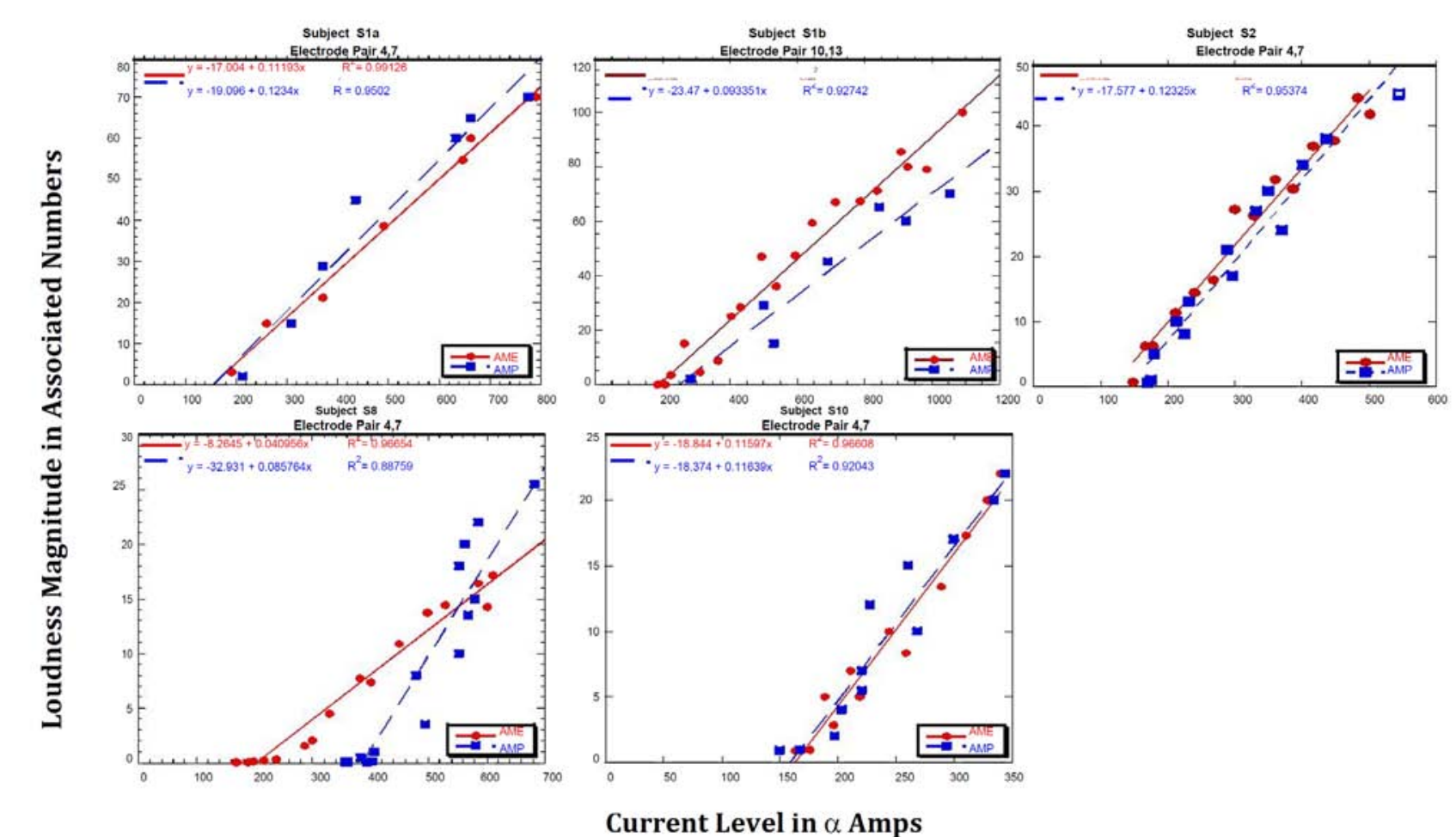

Figure 2: Examples of "linear-classified" loudness growth functions, based on visual observation, using AME and AMP data different subjects. Loudness in associated numbers is plotted versus current applied to the designated bipolar electrode pairs. 


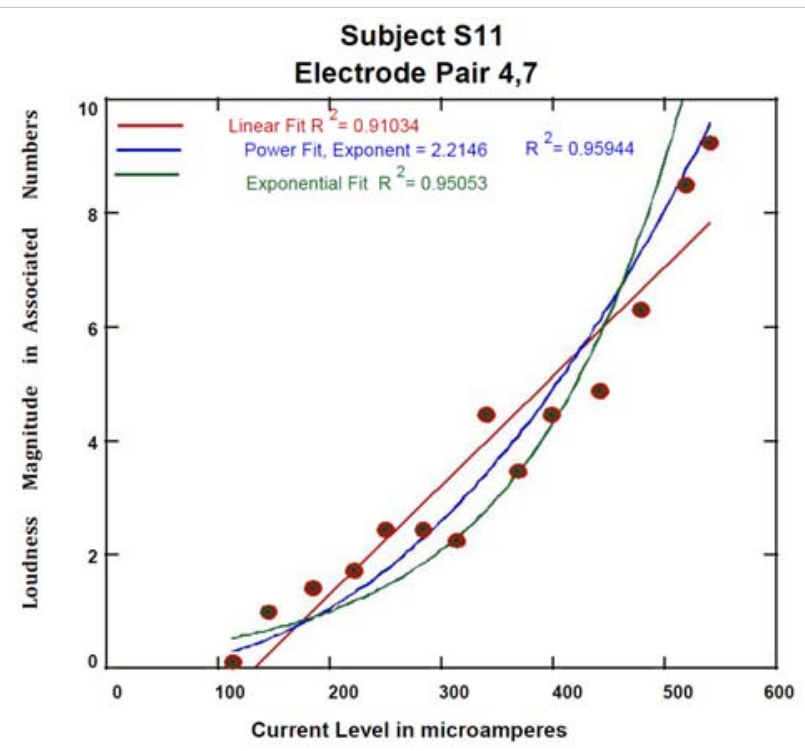

Figure 3: Loudness estimation using AME for Subject S11 on electrode pair 4,7 , fit with linear, power, and exponential functions. Note that $\mathrm{R}^{2}$ values of the three fit functions (linear, power, exponential) all exceed a value of 0.9

fit loudness growth functions were observed [Table 2].

Table 3 shows examples of the AICc best-fit function results measured on various electrode pairs for the prelinguallydeafened subjects [Table 3].

According to the results from the AICc analysis, the shape of the loudness growth functions were consistent with the classifications based on observation and indicated that there were two classes of growth functions; linear and expansive (power and exponential best fit functions). Subjects S1a, and S1b, S2, S8, and S10 seem to demonstrate best-fit linear loudness functions, whereas subjects S3, S4, S5, S6, S7, S9, S11, and S12 mostly demonstrate best-fit expansive functions (power, exponential) Subjects were classified as having "linear" or "expansive" based on the modal score or the most frequent best-fit functions measured on multiple electrode pairs for that individual. Some subjects with expansive growth of loudness demonstrate both power and exponential best-fit functions on different electrode pairs, as shown by Subject S3 in Table 2.

\section{Effects of Electrode Separation on the shape of the growth of loudness functions.}

Preliminary data was obtained to study the effect of electrode separation in bipolar stimulation on the growth of loudness functions for these different classes of subjects. Figure 4 shows growth of loudness functions for different electrodes and different spatial separations between the stimulating "active" and "return" electrodes for bipolar pairs for two subjects (Figure 4).

Figure 4 (left) demonstrates linear functions for all electrode pairs and electrode separations with an increasing slope value for wider bipolar configuration for Subject S1a. Results in figure 4 (right) are shown for another subject (S3) demonstrating more "expansive" growth of loudness functions for different electrode pairs and electrode separations. Similar to Subject S1a, this subject demonstrates an increasing exponent value and a decrease in dynamic range as for wider bipolar stimulation, similar to results found in Chatterjee [4]. These preliminary data reveal that the general shape of the growth of loudness function is independent of stimulus configuration, but the exponent or slopes of the loudness growth functions are dependent on electrode separation, with wider bipolar separation producing steeper curves. The steeper curves are presumably produced because more nerve fibers are recruited as electrode separation increases but this effect was not studied in detail.

\section{Differences between AME and AMP slopes and exponents}

The loudness growth functions obtained via AMP methods generally yielded steeper slopes or higher exponents than for results obtained with AME measured on the same electrode pair, demonstrating the "regression effect," that describes the phenomenon in which subjects typically reduce the range of the variable that they control. This phenomenon has been commonly observed in previous studies using these methods $[26,14]$.

Loudness growth functions that were previously measured using magnitude estimation have shown that loudness growth may have been best fit by a power function with an approximate exponent of 2.72 for bipolar electrode pairs [12]. For comparison to this study, we fit our data with power-fit functions and obtained exponents for all the subjects for $\mathrm{BP}+2$ electrode pairs only, for the AME and AMP experiments separately, for all of the data, including those with linear and exponential best fits. Analysis was done on only the $\mathrm{BP}+2$ electrode configuration due to above-mentioned effects of increased exponents with larger bipolar separations. The results for the $\mathrm{BP}+2$ bipolar pairs are shown in Figure 5. Subject S5 was not included in this data set because $\mathrm{BP}+2$ bipolar-paired electrode configurations were not measured on this subject in these experiments (Figure 5).

The mean power-function exponent for loudness functions best-fit by power functions, based on the AICc analysis was approximately 2.47 for AME experiments only, similar to the exponent 2.72 reported by $\mathrm{Fu}$ and Shannon [12]. The mean power-function exponent obtained for only the classified best-fit power functions, based on the AICc analysis for AMP experiments, was approximately 3.05. The histograms in figure 5 (AME results shown on the left, AMP shown on the right) reveal that when the AICc obtained best-fit functions were not power-functions, there were larger distributions of exponents, as shown by the histograms in Fig. 5, the exponents were generally higher for exponential best-fit functions (mean AME exponent $=4.74$; mean AMP exponent $=5.34$ ) and lower for linear best-fit functions closest to an exponent of around 1, (mean AME exponent = 1.266; mean AMP exponent $=1.16)$. The mean exponents $(+/-1$ s.d.) for power-fit functions fit to AICc -classified linear, power, and exponential best-fit functions and standard deviations were plotted in figure 6 for the AME (left) and AMP (right experiments). There was some overlap in the distributions between the 
The Growth of Loudness in Cochlear Implant Listeners; Possible Developmental Effects?

Table 2: AICc Analysis Results for Postlingually-deafened Subjects

\begin{tabular}{|c|c|c|c|c|c|c|c|c|c|}
\hline \multirow{2}{*}{ Subject } & \multirow{2}{*}{$\begin{array}{l}\text { Electrode } \\
\text { Pair }\end{array}$} & \multicolumn{3}{|c|}{ AICc values } & \multirow{2}{*}{ Models } & \multirow{2}{*}{$\begin{array}{c}\text { AICc } \\
\text { Difference }\end{array}$} & \multirow{2}{*}{$\begin{array}{l}\text { Probability } \\
\text { Correct in } \\
\text { Percent }\end{array}$} & \multirow{2}{*}{ Best Fit Model } & \multirow{2}{*}{$\begin{array}{l}\text { Power Function } \\
\text { Fit Exponent }\end{array}$} \\
\hline & & Linear & Power & Exponential & & & & & \\
\hline \multirow[t]{3}{*}{ S3 } & 10,13 & 36.32 & 5.91 & 2.55 & lin/pow & 30.4 & $0.01 / 99.9$ & Power & 6.427 \\
\hline & & & & & pow/exp & 3.36 & $15.71 / 84.29$ & Exponential & \\
\hline & & & & & $\operatorname{lin} / \exp$ & 33.77 & $0.01 / 99.9$ & Exponential & \\
\hline \multirow[t]{3}{*}{ S3 } & 16,19 & 109.61 & 88.62 & 95.61 & lin/pow & 20.99 & $0.01 / 99.9$ & Power & 3.62 \\
\hline & & & & & pow/exp & -6.99 & $97.07 / 2.93$ & Power & \\
\hline & & & & & $\operatorname{lin} / \exp$ & 13.99 & 0.09 / 99.91 & Exponential & \\
\hline \multirow[t]{3}{*}{ S4 } & 10,13 & 36.32 & 5.91 & 2.55 & lin/pow & 30.4 & $0.01 / 99.99$ & Power & 5.207 \\
\hline & & & & & pow/exp & 3.36 & $15.71 / 84.29$ & Exponential & \\
\hline & & & & & $\operatorname{lin} / \exp$ & 33.77 & $0.01 / 99.99$ & Exponential & \\
\hline \multirow[t]{3}{*}{ S4 } & 4,7 & 27.56 & -2.57 & -0.2 & lin/pow & 30.13 & $0.01 / 99.99$ & Power & 3.617 \\
\hline & & & & & pow/exp & -2.37 & $76.08 / 23.92$ & Power & \\
\hline & & & & & $\operatorname{lin} / \exp$ & 27.76 & $0.01 / 99.99$ & Exponential & \\
\hline \multirow[t]{3}{*}{ S5 } & 12,14 & 80.53 & 61.94 & 64.73 & lin/pow & 18.59 & $0.01 / 99.99$ & Power & 2.655 \\
\hline & & & & & pow/exp & -2.79 & $80.11 / 19.89$ & Power & \\
\hline & & & & & $\operatorname{lin} / \exp$ & 15.8 & $0.04 / 99.96$ & Exponential & \\
\hline \multirow[t]{3}{*}{ S6 } & 4,7 & 44.21 & 29.38 & 25.59 & lin/pow & 14.83 & 0.06 / 99.94 & Power & 4.046 \\
\hline & & & & & pow/exp & 3.79 & $13.06 / 86.94$ & Exponential & \\
\hline & & & & & $\operatorname{lin} / \exp$ & 18.62 & $0.01 / 99.99$ & Exponential & \\
\hline \multirow[t]{3}{*}{ S6 } & 10,13 & 126.47 & 117.9 & 116.12 & lin/pow & 8.57 & 1.36 / 98.64 & Power & 5.552 \\
\hline & & & & & pow/exp & 1.78 & $29.18 / 70.82$ & Exponential & \\
\hline & & & & & $\operatorname{lin} / \exp$ & 10.35 & $.56 / 99.44$ & Exponential & \\
\hline \multirow[t]{3}{*}{ S7 } & 10,13 & 34 & 15.89 & 13.78 & lin/pow & 18.11 & $0.01 / 99.9$ & Power & 4.23 \\
\hline & & & & & pow/exp & 2.11 & $25.88 / 74.12$ & Exponential & \\
\hline & & & & & $\operatorname{lin} / \exp$ & 20.22 & $0.01 / 99.9$ & Exponential & \\
\hline \multirow[t]{3}{*}{ S7 } & 4,7 & 46.9 & 38.44 & 40.99 & lin/pow & 8.46 & $1.43 / 98.57$ & Power & 3.225 \\
\hline & & & & & pow/exp & -2.55 & $78.19 / 21.81$ & Power & \\
\hline & & & & & $\operatorname{lin} / \exp$ & 5.91 & $4.95 / 95.05$ & Exponential & \\
\hline \multirow[t]{3}{*}{ S9 } & 10,13 & 36.32 & 5.91 & 2.55 & lin/pow & 30.4 & $0.01 / 99.99$ & Power & 6.91 \\
\hline & & & & & pow/exp & 3.36 & $15.71 / 84.29$ & Exponential & \\
\hline & & & & & $\operatorname{lin} / \exp$ & 33.77 & $0.01 / 99.99$ & Exponential & \\
\hline \multirow[t]{3}{*}{ S11 } & 4,7 & 0.14 & -8.36 & -13.7 & lin/pow & 8.5 & 2.35 / 97.65 & Power & 2.441 \\
\hline & & & & & pow/exp & 5.34 & 93.92 / 6.08 & Exponential & \\
\hline & & & & & $\operatorname{lin} / \exp$ & 13.84 & $.16 / 99.84$ & Exponential & \\
\hline \multirow[t]{3}{*}{ S12 } & 16,19 & -1.29 & -24.73 & -6.04 & lin/pow & 23.44 & $0.01 / 99.99$ & Power & 2.3 \\
\hline & & & & & pow/exp & -18.69 & 93.30 / 6.70 & Power & \\
\hline & & & & & $\operatorname{lin} / \exp$ & 4.75 & $0.01 / 99.99$ & Exponential & \\
\hline \multirow[t]{3}{*}{ S12 } & 10,13 & -10.7 & -27.67 & -4.35 & lin/pow & 16.97 & 0.02 / 99.98 & Power & 2.017 \\
\hline & & & & & pow/exp & -23.32 & 99.99 / 0.01 & Power & \\
\hline & & & & & $\operatorname{lin} / \exp$ & -6.35 & $96.00 / 4.0$ & Linear & \\
\hline
\end{tabular}

expnents for the different best-fit models as shown in figure 5.

Figure 6 shows the mean power-fit exponent for all linear, power, and exponential best-fit functions as determined by the AICc analysis, with +/- standard deviation error bars. Single factor
ANOVA analysis revealed a significant difference in the power-fit function exponents between all groups $(F(2,42)=34.24, p<0.05)$ for the AME experiments, as well as for the AMP experiments $(f(2,28)=39.85, p<0.05)$. Significant differences were confirmed by the Bonferroni and Fischer's Exact test. 
Absolute Magnitude Estimation for Loudness and Numbers on Indicated Electrodes Subject S1a
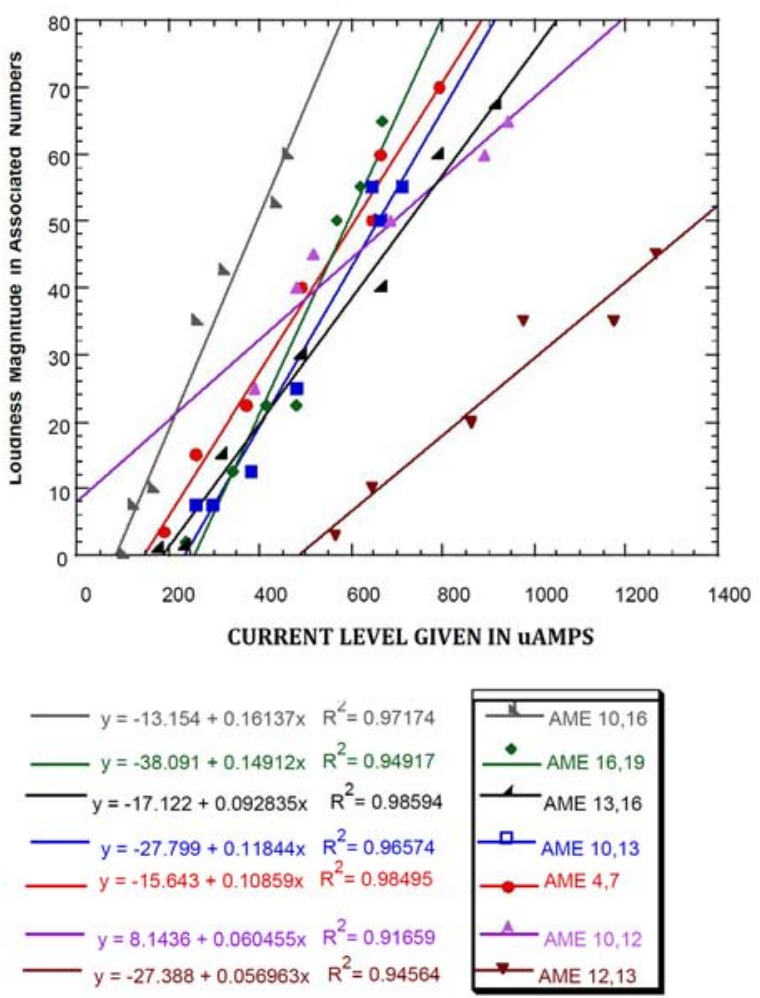

Absolute Magnitude Estimation for Loudness

and

Numbers on Indicated Electrodes

Subject \$3

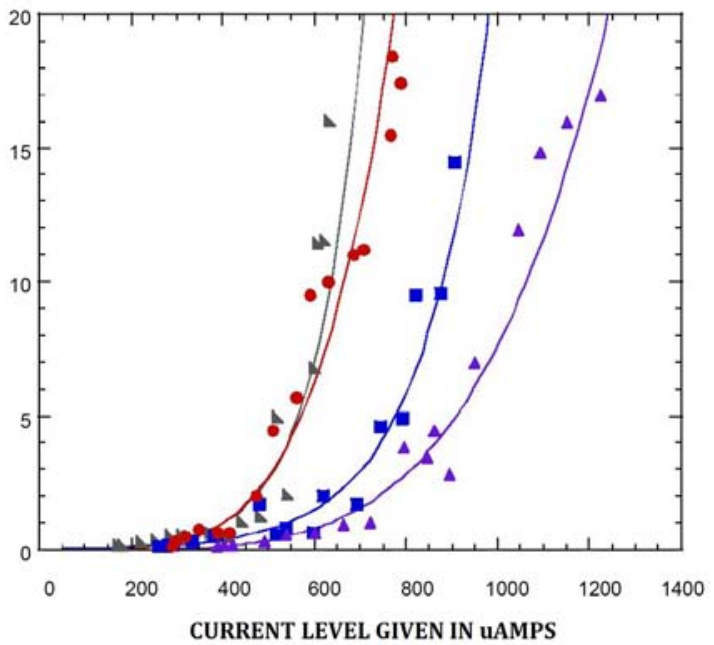

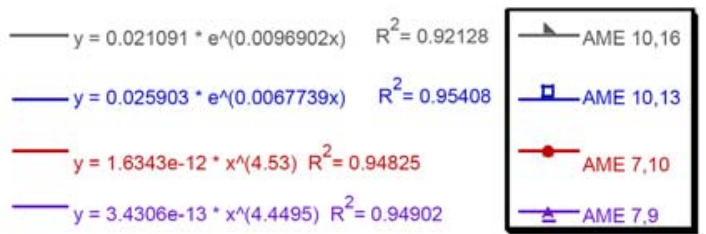

Figure 4: Absolute Magnitude Estimation between loudness and numbers for Subject S1a and Subject S3 on various electrode pairs and for various electrode separations.
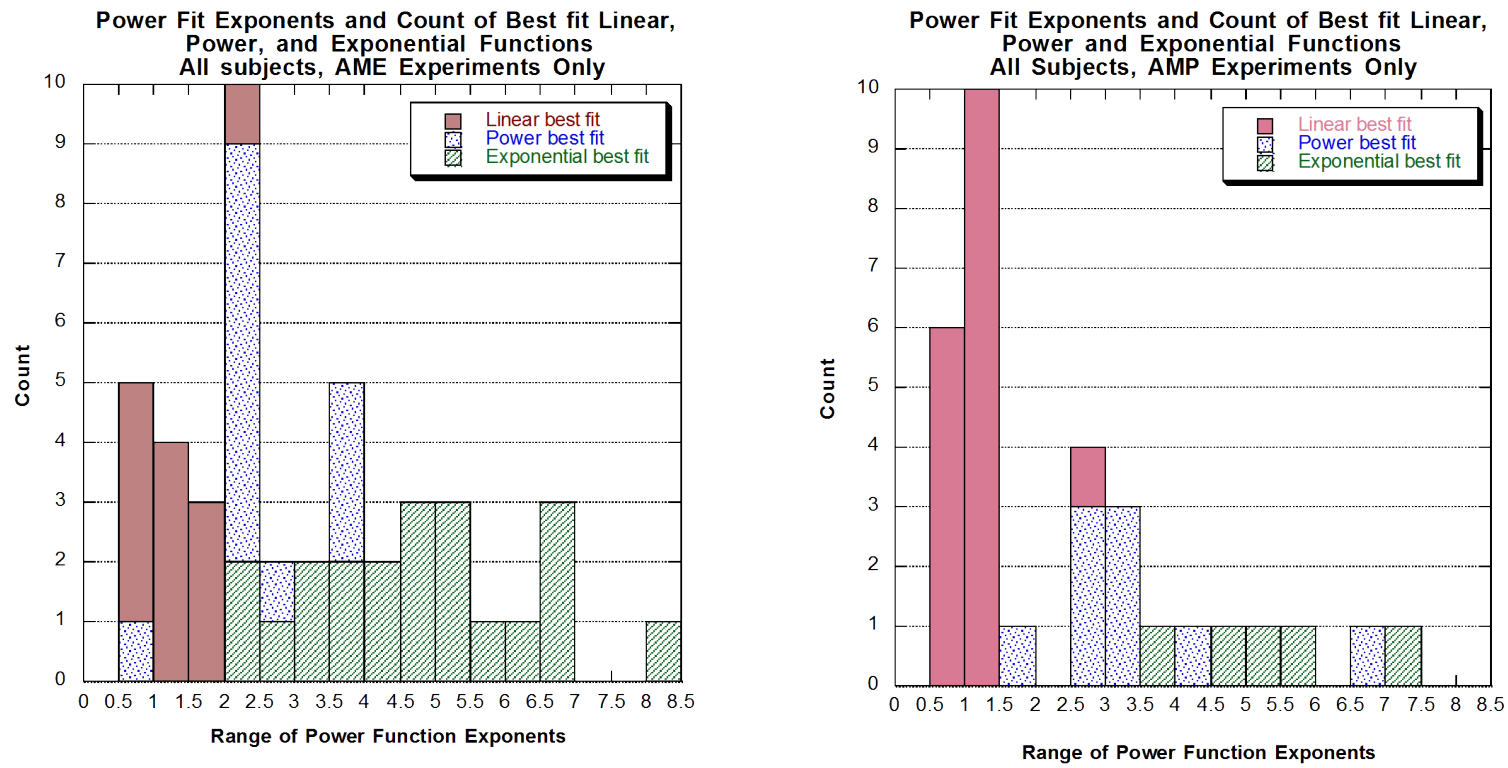

Figure 5: Distributions of power-fit function exponents for all AICc best fit functions for AME experiments (left), and AMP experiments (right), including $\mathrm{BP}+2$ electrode configurations only for all subjects.

Citation: Anzalone NM, Smith RL (2017) The Growth of Loudness in Cochlear Implant Listeners; Possible Developmental Effects?. Int Open Acce Otolary 1(1): 16 

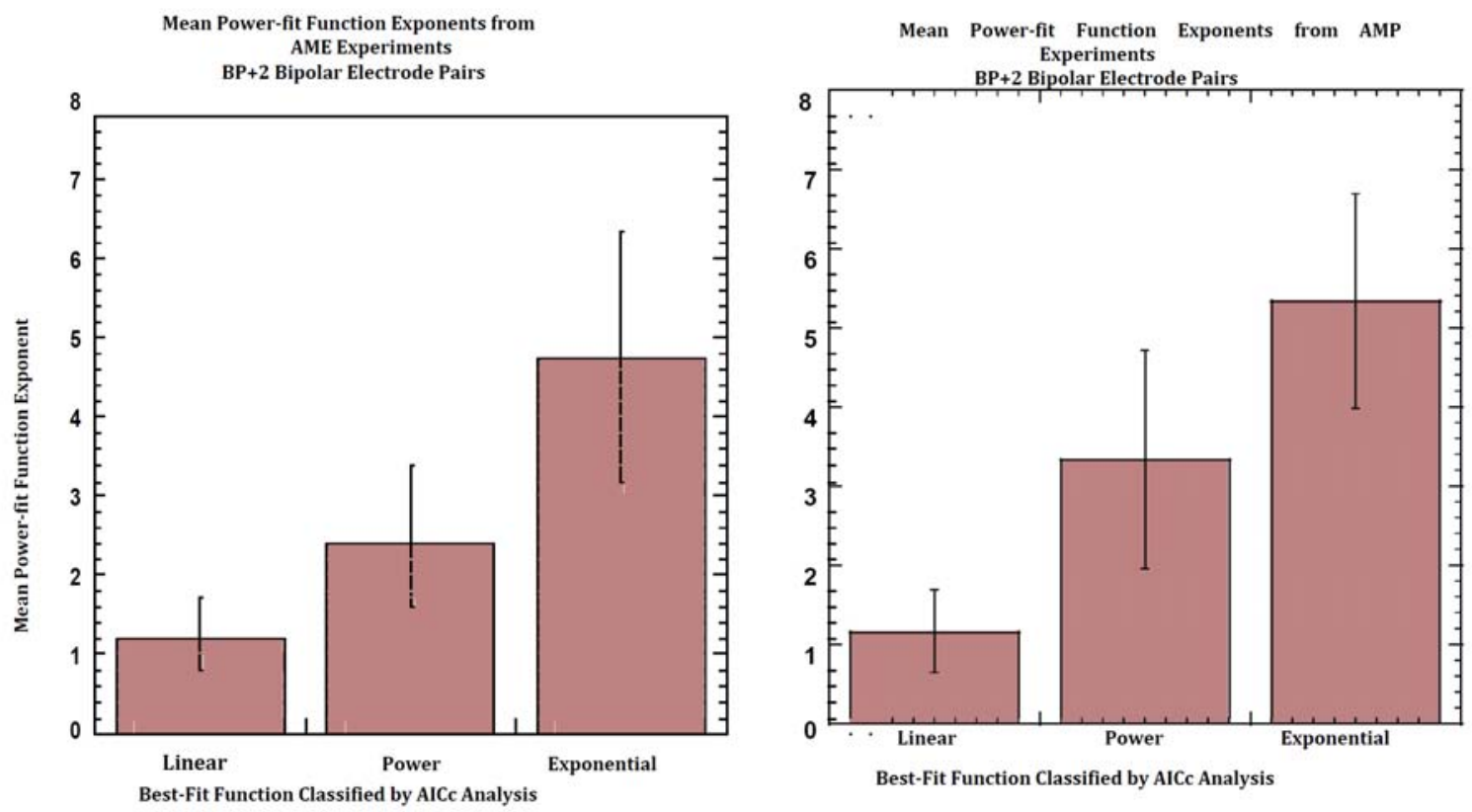

Figure 6: Shows the mean power-fit exponents for all loudness growth functions best-fit by linear, power, and exponential functions as determined by lowest AICc values. Results for AME experiments shown on the left and AMP results shown on the right. Single factor ANOVA analysis revealed a significant difference in the power-fit exponentials between all AICc classified best-fit function groups for both AME $(F(2,42)=34.24$, $p<0.05)$ and AMP exponents $(F(2,28)=39.85 . p<0.05)$.

\section{Power-function Exponents for Pre-and Post-lingual Subject Groups}

Subjects were grouped into two classes of subjects (prelingually-deafened and postlingually-deafened) based on subjects' etiology, obtained by the subjects' questionnaire answers about their hearing loss. A mean power-function exponent was obtained for all of the loudness growth functions for the prelingually-deafened and postlingually-deafened groups of subjects and was compared. These values included results from best-fit linear, power, and exponential loudness growth function data sets for several $\mathrm{BP}+2$ electrode pairs. The bar plots in figure 7 show the mean power exponents for the loudness growth functions on the various BP+2 electrode pairs for each subject. In each plot, the prelingually-deafened and postlingually-deafened subjects are divided by a thick black line. The AME results are shown in the left plot and the AMP results on shown in the right bar plot (Figure 7).

The mean power-function exponents obtained using the AME method, shown in figure 7 (left), for prelingual subjects $(M=1.68$, $S D=1.08, N=5)$ and figure 7 (right) - postlingual subjects $(M=4.22$, $S D=1.61, N=7$ ), were significantly different and lower for the prelingual group of subjects, as revealed by two-sample t-test assuming unequal variances $(t(10)=3.27, p<0.05)$. The mean power-function exponents obtained using the AMP method, as shown in the right panel of figure 8 for prelingual subjects $(M=1.24, S D=0.95, N=5)$ and post-lingual subjects $(M=3.069$, $S D=1.62, N=7)$ were also significantly different $(t(10)=2.45$, $p<0.05)$, as shown by a two sample t-test assuming unequal variances. Results were confirmed by the Tukey HSD post-hoc analysis. The power function exponents were significantly lower for the prelingual group. The means and standard deviations are plotted for the prelingually-deafened versus the postlinguallydeafened subjects in Figure 8 for the AME experiments (left) and AMP experiments (right). Hence, when the power function is used to fit all loudness growth curves, loudness growth was significantly shallower for the pre-lingually deafened group, compared to the post-lingually deafened group (Figure 8).

\section{Effects of Dynamic Range and Threshold}

In a study by Zeng and Shannon, [32], it was suggested that the electrical loudness growth function and the steepness of the loudness growth function is dependent on the electrical dynamic range. It was also suggested that larger the dynamic range, the smaller the slope of the loudness growth function becomes and the gradual tail portion near threshold of the loudness growth function increases with increasing dynamic range on a linear scale of electrical stimulus level in microamperes [34].

The relationship between electrical dynamic range in current level (Amps) and the shapes of the loudness growth functions is shown in figure 9. The mean dynamic range is shown for each AICc best-fit function category (linear, power, and exponential) for the loudness growth function results obtained for the $\mathrm{BP}+2$ electrode pair configurations for all subjects and their dynamic ranges measured on those electrode pairs. The mean dynamic ranges were calculated for each electrode pair on which the loudness growth functions were measured, and were plotted in the bar graph, including the standard deviations as the error 
Table 3: AICc Analysis Results for Prelingually-deafened Subjects (AME Experiments only)

\begin{tabular}{|c|c|c|c|c|c|c|c|c|c|}
\hline \multirow[t]{2}{*}{ Subject } & \multirow[t]{2}{*}{$\begin{array}{l}\text { Electrode } \\
\text { Pair }\end{array}$} & & \multirow{2}{*}{\begin{tabular}{|c} 
AICc \\
values
\end{tabular}} & \multirow[b]{2}{*}{ Exponential } & \multirow[t]{2}{*}{ Models } & \multirow[t]{2}{*}{$\begin{array}{c}\text { AICc } \\
\text { Difference }\end{array}$} & \multirow[t]{2}{*}{$\begin{array}{l}\text { Probability } \\
\text { Correct in } \\
\text { Percent }\end{array}$} & \multirow[t]{2}{*}{ Best Fit Model } & \multirow[t]{2}{*}{$\begin{array}{c}\text { Power } \\
\text { Function Fit } \\
\text { Exponent }\end{array}$} \\
\hline & & Linear & & & & & & & \\
\hline \multirow[t]{3}{*}{ S1a } & & 24.92 & 38.86 & 39.48 & lin/pow & -13.94 & $99.91 / .09$ & Linear & \\
\hline & & & & & pow/exp & -0.62 & $57.63 / 42.37$ & Power & 0.951 \\
\hline & & & & & lin/exp & -14.56 & $99.93 / 0.07$ & Linear & \\
\hline \multirow[t]{3}{*}{ S1a } & 4,7 & 32.81 & 44.17 & 34.6 & lin/pow & -11.36 & $99.66 / .34$ & Linear & \\
\hline & & & & & pow/exp & 9.57 & $.82 / 99.18$ & Exponential & 1.853 \\
\hline & & & & & lin/exp & -1.79 & $70.85 / 29.15$ & Linear & \\
\hline \multirow[t]{3}{*}{ S1b } & 10,13 & 78.91 & 80.33 & 106.78 & lin/pow & -1.42 & $67.06 / 32.94$ & Linear & \\
\hline & & & & & pow/exp & -26.45 & 99.99 / 0.01 & Power & 0.779 \\
\hline & & & & & lin/exp & -27.87 & 99.99 / 0.01 & Linear & \\
\hline \multirow[t]{3}{*}{ S2 } & 10,13 & 41.95 & 45.87 & 44.37 & $\operatorname{lin} /$ pow & -4.22 & $87.67 / 12.33$ & Linear & \\
\hline & & & & & pow/exp & 1.5 & $32.15 / 67.85$ & Exponential & 1.243 \\
\hline & & & & & $\operatorname{lin} / \exp$ & -2.42 & $77.11 / 22.89$ & Linear & \\
\hline \multirow[t]{3}{*}{ S2 } & 10,13 & 22.94 & 26.11 & 38.02 & $\operatorname{lin} /$ pow & -3.16 & $83.03 / 16.97$ & Linear & \\
\hline & & & & & pow/exp & -11.91 & $99.74 / .26$ & Power & 0.8506 \\
\hline & & & & & lin/exp & -15.08 & $99.95 / 0.05$ & Linear & \\
\hline \multirow[t]{3}{*}{ S8 } & 4,7 & 13.29 & 14.86 & 33.03 & lin/pow & -1.57 & $68.74 / 31.26$ & Linear & \\
\hline & & & & & pow/exp & -18.17 & $99.99 / 0.01$ & Power & 1.331 \\
\hline & & & & & $\operatorname{lin} / \exp$ & -19.74 & $99.99 / 0.01$ & Linear & \\
\hline \multirow[t]{3}{*}{ S8 } & 4,7 & 33.82 & 37.61 & 47.65 & $\operatorname{lin} /$ pow & -3.791 & $86.94 / 13.06$ & Linear & \\
\hline & & & & & pow/exp & -10.04 & $99.34 / 0.66$ & Power & 1.067 \\
\hline & & & & & $\operatorname{lin} / \exp$ & -13.83 & $99.90 / 0.10$ & Linear & \\
\hline \multirow[t]{3}{*}{$\mathrm{S} 10$} & 10,13 & 13.29 & 14.86 & 33.03 & lin/pow & -1.57 & $68.74 / 31.26$ & Linear & \\
\hline & & & & & pow/exp & -18.17 & $99.99 / 0.01$ & Power & 2.025 \\
\hline & & & & & $\operatorname{lin} / \exp$ & -19.74 & $99.99 / 0.01$ & Linear & \\
\hline \multirow[t]{3}{*}{ S10 } & 16,19 & 15.85 & 18.55 & 22.95 & lin/pow & -2.7 & $79.45 / 20.55$ & Linear & \\
\hline & & & & & pow/exp & -4.4 & $90.19 / 9.81$ & Power & 1.661 \\
\hline & 10,13 & & & & lin/exp & -7.1 & $97.27 / 2.73$ & Linear & \\
\hline
\end{tabular}

AICc analysis results for prelingually-deafened subjects on different $\mathrm{BP}+\mathrm{N}$ electrode pairs from AME experiments. The best fit models based on lowest AICc values and probability that model correctly fits the data. Lowest AICc values and best-fit models are highlighted.

bars. A one-way ANOVA analysis between the means showed no significant difference between the dynamic ranges for the three best-fit function categories $(F(2,79)=1.29, p=0.297)$ measured on the $\mathrm{BP}+2$ electrode pairs (Figure 9).

It has been suggested that the sensation of threshold and loudness could be related to the physiological status at the electrode-neuron interface, i.e. the local neuron survival or the location of the electrode relative to the nerve [22], and possible morphological and physiological differences may exist between prelingual and postlingual patients [27]. Mean thresholds for all of the $\mathrm{BP}+2$ electrode pairs were also compared between the AICc classified loudness growth function groups (linear, power, exponential) and between prelingual and postlingual subject groups. Figure 10 shows the relationship between the AICc bestfit linear, power, and exponential functions for AME obtained results for the $\mathrm{BP}+2$ electrode pair configurations for all subjects and their threshold values measured on the respective electrode pairs (Figure 10).

The mean thresholds that were measured on each of the $\mathrm{BP}+2$ electrode pairs on which the loudness best-fit functions (linear, power, and exponential) were obtained, and were plotted in the bar graph including the standard deviations as error bars. Mean thresholds measured in current $\mu$ Amps),level (were lower for the AICc-classified exponential best-fit functions than for the classified best-fit linear or power functions, but a single factor ANOVA analysis revealed that there was no significant difference between the threshold means $(F(2,79)=1.70, p=0.1877)$. Directly comparing the dynamic ranges (MAL - threshold) measured in current level ( $\mu \mathrm{Amps}$ ), for the prelingual versus postlingual groups revealed the mean dynamic ranges for prelingual subjects $(M=545.18, S D=285.36, N=5)$ were not significantly different from the dynamic ranges for postlingual subjects $(M=387.17, S D$ 

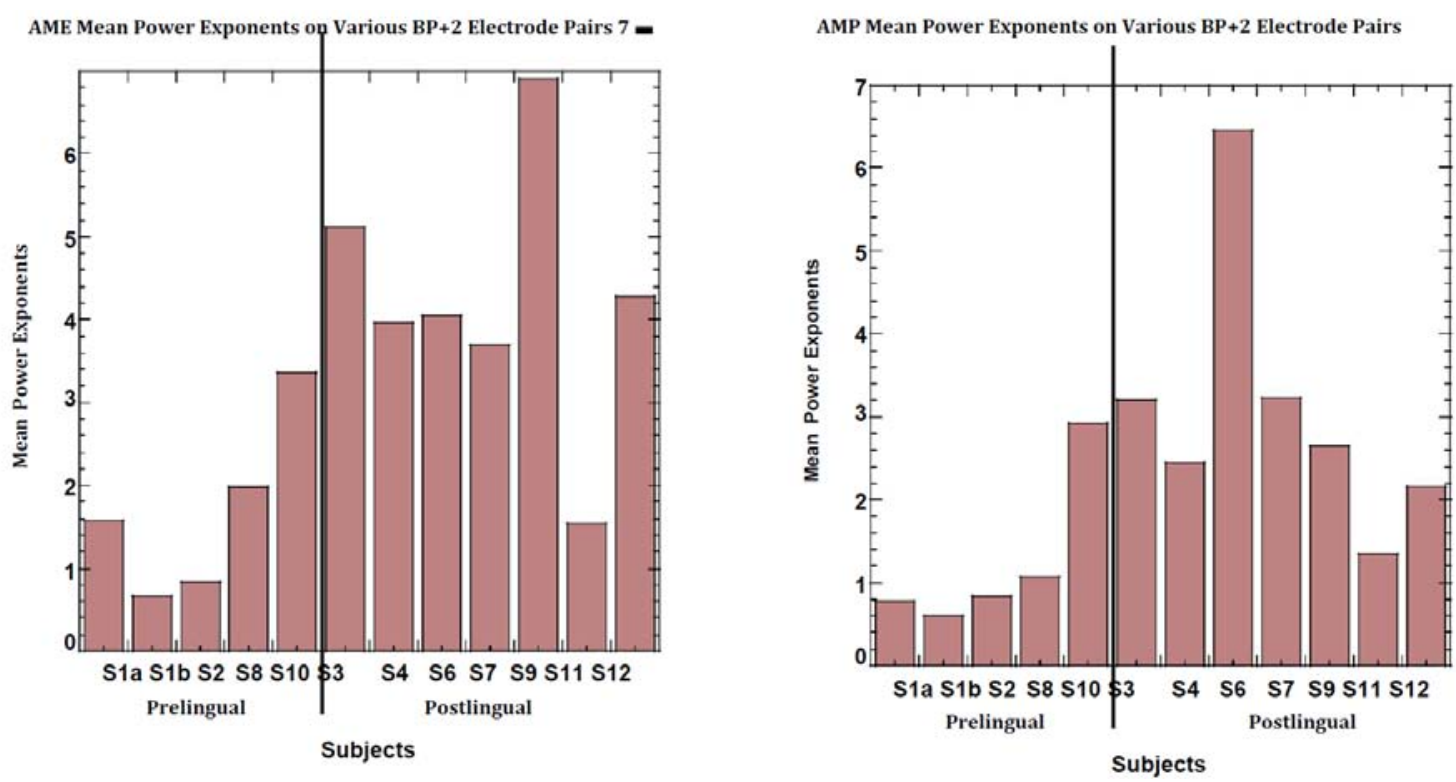

Figure 7: Mean power-fit exponents for all loudness growth functions measured on BP+2 electrode pairs for each subject, and shown for all prelingually- and post-lingually-deafened subjects. Error bars are not shown for each subject due to insufficient sample sizes.
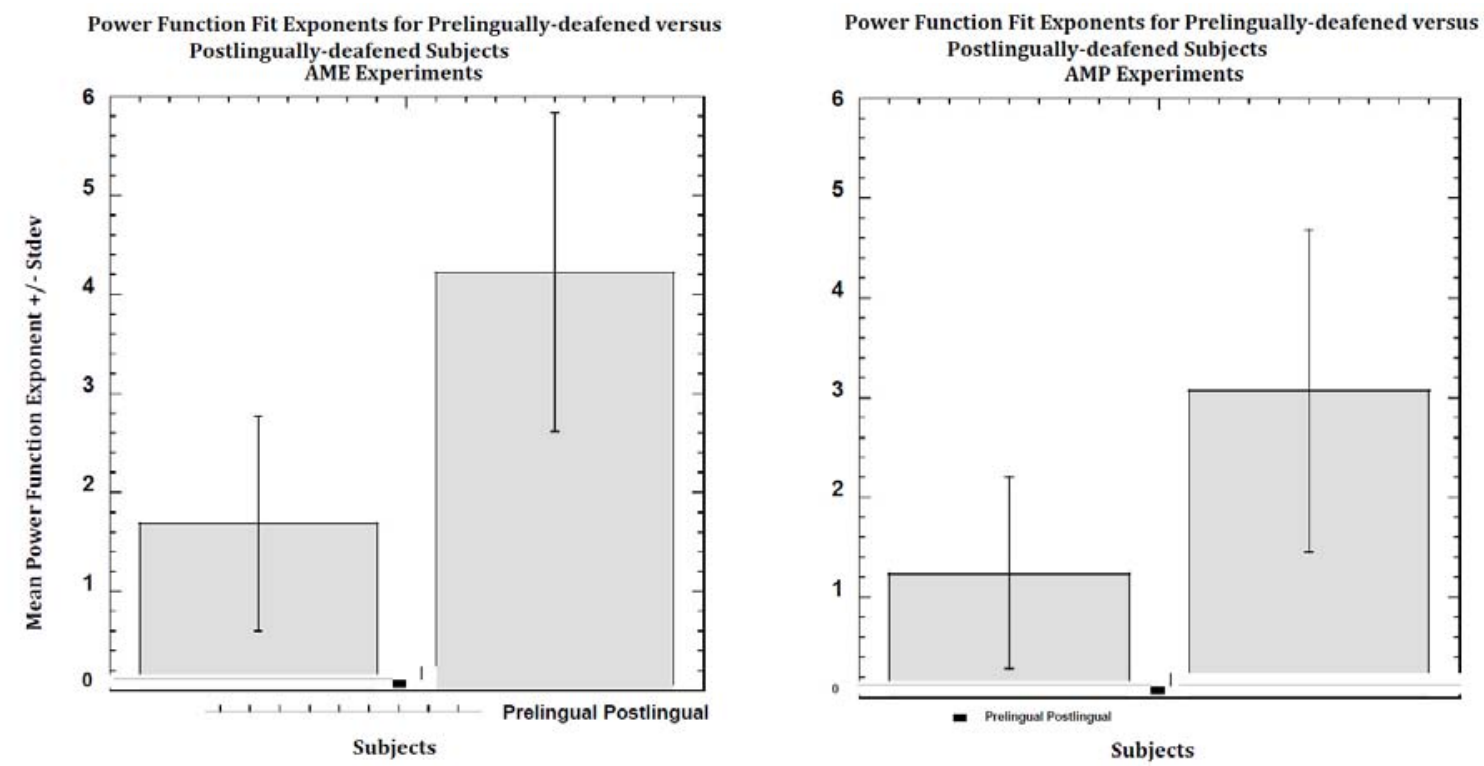

Figure 8: Mean power fit function exponents +/- standard deviations compared between prelingually- and postlingually-deafened subjects for AME (shown right) and AMP (left). Two sample t-tests revealed exponents were significantly lower for prelingually-deafened subjects for loudness functions obtained with AME $(\mathrm{t}(10)=3.27, \mathrm{p}<0.05)$ and AMP experiments $(\mathrm{t}(10)=2.45, \mathrm{p}<0.05)$.

$=114.62, N=7)$, as shown by two-sample t-test assuming unequal variances $(t(5)=1.17, p=0.29)$.

The mean thresholds for prelingual subjects $(M=191.75, S D$ $=27.01, N=5$ ) were not significantly different from the mean thresholds of postlingual subjects $(M=149.96, S D=89.29, N=7)$, $(t(8)=1.07, p=0.31)$, as shown by two-sample t-test assuming unequal variances.

\section{Discussion}

There were two primary categories of loudness growth functions observed in this study - linear and expansive, and the dichotomy appears to be subject dependent, demonstrating at least two different classes of cochlear implant users; those who exhibit primarily linear loudness growth functions and those that can be best-approximated by a expansive best-fit function 


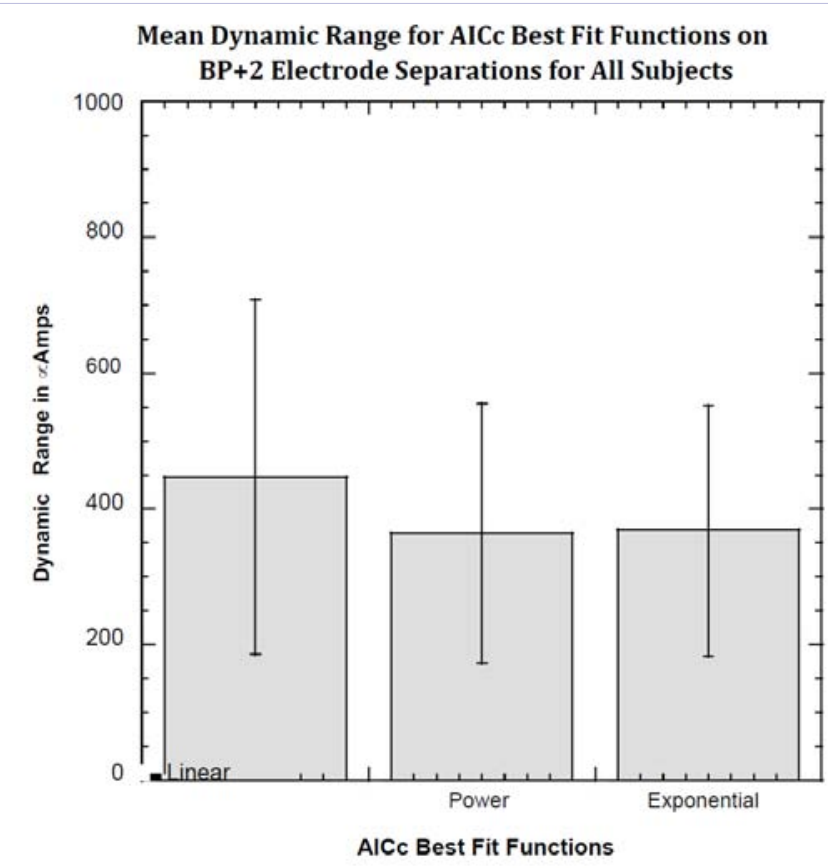

Figure 9: Mean dyamic ranges +/- standard deviations corresponding to AICc linear, power, and exponential best-fit functions measured on $\mathrm{BP}+2$ electrode configurations for all subjects. The shape of the gowth of loudness functions are independednt of dynamic range, as revelad by a single-factor ANOVA analysis, showing no significant difference in dynamic ranges between groups $(\mathrm{F}(2,79)=1.29, \mathrm{p}=0.297)$.

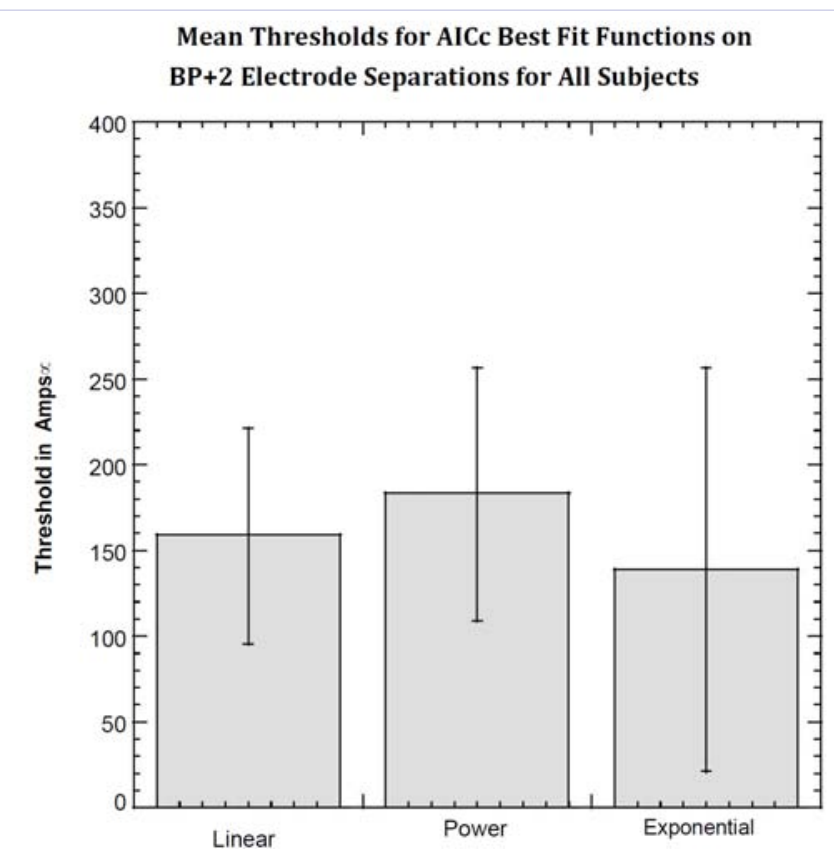

Figure 10: Mean threshold values +/- standard deviations corresponding to AICc linear, power, and exponential best-fit functions measured on $\mathrm{BP}+2$ electrode configurations for all subjects. The shape of the growth of loudness functions are independent of threshold values, as revealed by a single-factor ANOVA analysis, showing no significant difference in threhsolds between groups $(F(2,79)=1.70, p=0.29)$. (including the power function and exponential function). The primary difference between the clinical history of two groups of subjects that fall into the "linear" and "expansive" loudness function classes is that the subjects that show mostly linear functions fall into the prelingually-deafened class and the "expansive" loudness function group is mostly postlinguallydeafened. Hence the data suggest that the shape of the loudness functions is associated with the subject's listening experience and age of implantation.

There were no significant differences of dynamic range or thresholds accross all subjects' growth of loudness functions using the same electrode configuration.

The above results can be seen by visually observing and comparing the measured growth of loudness functions. In addition, the AICc method of modeling is effective for objectively analyzing and comparing growth of loudness functions and agrees with the perhaps more subjective observations. Best-fit loudness growth functions as determined by AIC appear to show a difference in the loudness growth functions across subjects and across electrode pairs within individual subjects. The range of functions observed is greater than previously reported and goes from linear to expansive, suggesting that individual variations in loudness growth should be incorporated in the design of cochlear implant sound processors. Our results reveal that some subjects may show best-fit linear, power, and exponential functions on different electrode pairs and different individuals can show a range of these best-fit functions. By accurately identifying the shape of the loudness growth functions for different subjects, it should be possible to modify the shape of the mapping functions to restore or attempt to optimize normal growth of loudness for individual cochlear implant users.

Results of the power-fit function exponents also revealed that there is a relationship between the exponents of the loudness functions and the two subject populations (pre-lingual vs. postlingual), showing that pre-lingual subjects may generally show significantly lower growth of loudness function exponents when fit with power-functions.

Loudness growth functions that were previously measured using magnitude estimation have shown that loudness growth may have been best fit by a power function with an approximate exponent of 2.72 [12]. Our results reveal that the exponent of the power-fit function, when proven as the best-fit function, agrees with previously obtained approximate exponents. However, when a power-fit function is fit to a best-fit linear or exponentialfunction, the exponents reveal a much wider distribution of values, approximating a value near 1 for linear functions and a value close to 5 for exponential functions. In Shannon, et al. 2004, [23] and Fu and Shannon 1998,[12] it was predicted that a cross-modal match between the best-approximate loudness function exponents of normal hearing listeners (0.6) and the approximated loudness function exponent of the cochlear implant listeners (2.72), by taking the ratio of the two exponents, revealed that the exponent resulting from the ratio of the two agreed with the peak in the speech intelligibility performance of 
cochlear implant listeners. All of the subjects in their experiments were postlingually-deafened and our data agrees with the bestfit loudness growth function exponent range obtained on the postlingually-deafened subjects in this study. However, the few prelingually-deafened subjects in our study show best-fit approximate exponents around 1, demonstrating a much more linear loudness growth function. These results may suggest that an appropriate cross-modal match between acoustic and electric amplitude to obtain more normal loudness growth may be different for the prelingually-deafened population of subjects.

An exponential loudness growth model proposed by Zeng and Shannon [28] assumes that the loudness growth function can be predicted by determining threshold and uncomfortable loudness levels, but our preliminary results suggest that the shape of the loudness growth function is independent of electrical dynamic range and thresholds for the $\mathrm{BP}+2$ electrode pairs.

The results suggested that there were no significant differences in thresholds or dynamic ranges between prelinguallyand postlingually-deafened subjects. These results may be due to the large variability found in thresholds and dynamic ranges measured on several $\mathrm{BP}+2$ electrode pairs within individual subjects as well as between subjects. In this study, results suggest that there may be two populations of cochlear implant listeners: those who have "developed" the expansive nonlinearities in the central auditory system and those, mainly early-deafened subjects implanted at four or more years after birth, who have not "developed" the expansive loudness growth in the central auditory system. It should be noted, however, that prelinguallydeafened subjects with early implantation - before 3 years of age, were not included in this study. Since these results show that the shapes of the loudness functions vary both across subjects, as shown by the "linear" and "expansive" best-fit classifications, and within an individual subject on each electrode pairs, as shown by individuals displaying both power and exponential best-fit functions. Since current cochlear implant speech processors assume a uniform loudness growth function for all electrodes and subjects, it may be indicated that speech processors may need to be redesigned to take into account individual differences in loudness growth to maximize listening performance for cochlear implant listeners.

There are limited previous studies performed on loudness growth functions and speech recognition in prelinguallydeafened subjects. Early-deafened patients may have limited auditory experience and due to long-term sound deprivation prior to cochlear implantation, these patients may have poorer abilities of speech recognition as well as differences in psychophysical function [3, 27]. Although speech recognition abilities for the subjects in this study were not measured, there may be a possible relationship between speech recognition abilities for prelingually-deafened subjects who have a lack of early normal speech listening experience, and improper amplitude transformation between acoustic and electrical stimulation. These variables will be addressed further in future investigations.

\section{Acknowledgements}

We thank Monita Chatterjee and Mark Robert and the House Ear Institute for their generous help and equipment loan, and Cochlear Corporation for providing software, equipment, and advice. Thanks to Mohamed Bingabr for his dedication to programming and getting the NIC system up and running. Thanks to Robert Ploutz-Snyder for his statistical advice and Rohit Subramanian for his help with the time-consuming data analysis. Thanks to Jozef Zwislocki for his helpful advice with the experimental methodology. Very special thanks to the anonymous subjects who spent many hours diligently working with us This work was supported by the Jim and Arlene Gerber fund for auditory research at the Institute for Sensory Research, and funds from the College of Engineering and Computer Science at Syracuse University.

\section{References}

1. Boex CS, Eddington DK, Noel VA, Rabinowitz WM, Tierney J and Whearty WE. Restoration of normal loudness growth for CIS sound coding strategies. Abstracts of 1997 Conference on Implantable Auditory Prostheses. 1997;26.

2. Boex CS, Pelizzone M, Piloux V and Montandon P. Use of loudness scaling measurements to determine compressive mapping in speech processing for cochlear implants. Abstracts of 1995 Conference on Implantable Auditory Prostheses. 1995;57.

3. Busby PA, Tong YC, and Clark GM. Psychophysical studies using a multiple-electrode cochlear implant in patients who were deafened early in life. Audiology. 1992;31(2):95-111.

4. Chatterjee M. Effects of stimulation mode on threshold and loudness growth in multi electrode cochlear implants. J Acoust Soc Am. 1999;105(2):850-860.

5. Collins AA and Gescheider GA. The measurement of loudness in individual children and adults by absolute magnitude estimation and cross-modality matching. J Acoust Soc Am. 1989;85(5):2012-2021.

6. Cosendai G and Pelizzone M. Effects of the acoustical dynamic range on speech recognition with cochlear implants. Audiology. 2001;40(5):272-281.

7. Drullman, R. Speech intelligibility in noise: relative contribution of speech elements above and below the speech level. J Acoust Soc Am. 1995;98(3):1796-1798.

8. Eddington DK, Boex SpanoC, Tierney J, Noel V, Whearty M. Speech processors for auditory prostheses. Seventh Quarterly Progress Report, NIH Contract N01-DC-6-2100, 1997.

9. Freyman RL and Nerbonne GP. The importance of consonant-vowel intensity ratio in the intelligibility of voiceless consonants. J Speech Hear Res. 1989;32(3):524-535.

10. Freyman RL and Nerbonne GP and Cote HC. Effect of consonantvowel ratio modification on amplitude envelope cues for consonant recognition. J Speech Hear Res. 1991;34(2):415-426.

11. Fu QJ. Loudness growth in cochlear implants: effect of stimulation rate and electrode configuration. Hear Res. 2005;202(1-2):55-62. doi:10.1016/j.heares.2004.10.004

12. Fu QJ and Shannon RV. Effects of amplitude nonlinearity on speech recognition by cochlear implant users and normal-hearing listeners. J Acoust Soc Am. 1998;104(5):2570-2577. 
13. Levitt H. Transformed up-down methods in psychoacoustics. J Acoust Soc Am. 1971;49(2):467-477.

14. Marks LE. Sensory Processes: The New Psychophysics. Academic Press, New York. 1974.

15. McKay CM. "Psychophysics and Electrical Stimulation in Cochlear Implants," In: F.-G. Zeng, A.N. Popper, and R.R. Fay (Eds.). Auditory Prostheses and Electric Hearing, Springer Verlag, NY; 2004: 286-333.

16. McKay CM, Remine MD and McDermott HJ. Loudness summation for pulsatile electrical stimuli of the cochlea: Effects of rate, electrode separation, level, and mode of stimulation. J Acoust Soc Am. 2001; 110(3):1514- 1524.

17. Motulsky HJ and Christopoulos A. Fitting models to biological data using linear and nonlinear regression. A practical guide to curve fitting. GraphPad Software Inc, San Diego CA. 2003.

18. Müller CG. Survey of cochlear implant work. J Acoust Soc Am Suppl. 1981;70(1):S52. doi: 10.1121/1.2018916

19. Pitt MA, Myung IJ and Zhang S. Toward a method of selecting among computational models of cognition. Psychological Review. 2002;109(3):472-491.

20. Plomp R. The negative effect of amplitude compression in multichannel hearing aids in the light of the modulation-transfer function. J Acoust Soc Am. 1988;83(6):2322-2327.

21. Sanpetrino NM and Smith RL. The growth of loudness functions measured in cochlear implant listeners using absolute magnitude estimation and compared using Akaike's Information Criterion. Paper in: Engineering in Medicine and Biology Society, EMBS $28^{\text {th }}$ Annual International Conference of the IEEE, New York. 2006;1:1642-1644. doi:10.1109/IEMBS.2006.259984

22. Shannon RV. Threshold and loudness functions for pulsatile stimulation of cochlear implants. Hearing Research. 1985;18(2):135 143

23. Shannon RV, Fu QJ, Galvin JJ III and Friesen L. Speech perception with cochlear implants, Chapter 8 in Auditory Prostheses, Zeng FG, Popper AN and Fay RR (Eds.), Springer Handbook of Auditory Research, Vol X, Springer-Verlag, New York, 2004;20:334-376.

24. Shannon RV, Zeng FG, Wygonski J, Kamath V and Ekelid M. Speech recognition using only temporal cues. In: the Auditory Processing of Speech: from Sounds to Words, Schouten MEH (Ed.), Mouton de Gruyter, Berlin. 1995;270(5234):303-304.

25. Stevens SS. Psychophysics: Introduction to its Perceptual, Neural, and Social Prospects, edited by G Stevens. Wiley, New York. 1975;99-133.

26. Stevens SS and Greenbaum HB. Regression effect in psychophysical judgment. Perception \& Psychophysics. 1966;1(5):439-446. doi:10.3758/BF03207424

27. Tong YC, Busby PA, Clark GM. Perceptual studies on cochlear implant patients with early onset of profound hearing impairment prior to normal development of auditory, speech, and language skills. J Acoust Soc Am 1988;84(3):951-962.

28. Zeng FG and Galvin JJ $3^{\text {rd }}$. Amplitude mapping and phonemerecognition in cochlear implant listeners. Ear \& Hearing. 1999;20(1):60-74.

29. Zeng FG, Grant G, Niparko J, Galvin J, Shannon R, Opie J, et al. Speech dynamic range and its effect on cochlear implant performance. J. Acoust. Soc. Am. 2002;111(1):377-386

30. Zeng FG and Shannon RV. Loudness balance between acoustically and electrically stimulated ears. Hear Res 1992;60:231-235.

31. Zeng FG and Shannon RV. Loudness- coding mechanisms inferred from electric stimulation of the human auditory system. Science. 1994;264(5158):564-566. doi: 10.1126/science.8160013

32. Zeng FG \& Shannon RV. Loudness of simple and complex stimuli in electric hearing. Annals of Otology Rhinology \& Laryngology, Suppl. 1995;166:235-238.

33. Zeng FG, Shannon RV and Hellman WS. Physiological processes underlying psychophysical laws," In: A.R. Palmer, A. Rees, A.Q. Summerfield, R. Meddis (Eds.), Psychophysical and Physiological Advances in Hearing, Proceedings of the $11^{\text {th }}$ International Symposium on Hearing. 1997;473-481.

34. Zeng FG and Shannon RV. Psychophysical laws revealed by electric hearing. NeuroReport. 1999;10(9):1931-1935.

35. Zwicker E and Scharf B. A model of loudness summation. Psychology Review. 1965;72:3-26.

36. Zwislocki JJ. Group and individual relations between sensation magnitudes and their numerical estimates. Perception \& Psychophysics. 1983;33(5):460-468.

37. Zwislocki JJ and Goodman DA. Absolute scaling of sensory magnitudes: A validation. Perception \& Psychophysics. 1980;28(1):28-38. 